\title{
COBORDISM PROPERTIES OF MANIFOLDS OF SMALL CATEGORY
}

\author{
M. V. MIELKE
}

Cobordism properties of certain manifolds $M$ with $C(M) \leqq 3(C(M)$ is the Lusternik-Schnirelman category of $M$ ) are studied (Lemma 1) with an application to "killing" homotopy groups by surgery (Theorem $\left.1^{*}\right)$.

An $n$-manifold is a smooth, connected, compact, $n$-dimensional manifold without boundary. "[ ]" denotes the "greatest integer" function.

THEOREM 1. If $M$ is $a[n / 3]$-connected $n$-manifold where $n \equiv 3 \bmod 4$ then $M$ cobounds the $n$-sphere $S^{n}$.

An equivalent statement of Theorem 1 is:

THEOREM 1*. If the homotopy groups of an $n$-manifold $M(n \equiv 3$ mod 4) can be killed by spherical modifications (surgery) through dimension $[n / 3]$ then all the homotopy groups through dimension $n-1$ can be so killed (cf. [5]).

Proof of Theorem 1. By $[1$, p. $52,199-200]$ any $[n / 3]$-connected $n$-manifold $(n>3)$ satisfies $C(M) \leqq 3$. In view of this and the fact that any 3 -manifold cobounds $S^{3}$ (cf. $[4$, p. 81]) the theorem follows from Lemma 1.

LEMMA 1. Let $M$ be an $n$-manifold with $C(M) \leqq 3$. If $n \equiv 3 \bmod 4$ then $M$ cobounds $S^{n}$.

Proof of Lemma 1. In view of [4] we may assume $n>3$. Let $W_{i} \in H^{i}\left(M ; Z_{2}\right)$ be the $i$ th Stiefel-Whitney class of $M$. The StiefelWhitney number associated to a tuple $i_{1}, \cdots, i_{r}$ where $i_{1}+\cdots+i_{r}$ $=n$ is defined to be $\left\langle W_{i_{1}} \cdots W_{i_{r}}, \mu\right\rangle$ where ${ }^{\alpha}\langle\rangle^{n}$ denotes the Kronecker index and $\mu \in H_{n}\left(M ; Z_{2}\right)$ is the fundamental class of $M$ (cf. [2]). By [4, p. 77] $M$ cobounds $S^{n}$ if and only if all StiefelWhitney numbers of $M$ are zero. To show $M$ cobounds $S^{n}$ it is sufficient, then, to show $W_{i_{1}} \cdots W_{i_{r}}=0$ for all tuples $i_{1}, \cdots, i_{r}$ satisfying $i_{1}+\cdots+i_{r}=0$.

A well-known result $([3$, p. 4$])$ on cup products is:

1. If $C(M) \leqq 3$ then the cup product of 3 or more positive dimensional cohomology classes is zero.

In view of this, Lemma 1 will be proved when it is shown that $W(i)=0$ for $i=0, \cdots, n$ where $W(i)=W_{i} W_{n-i}$.

Received by the editors July 21, 1968. 
The following results are needed for the proof. According to $\mathrm{Wu}$ [6] there exist cohomology classes $U_{r} \in \operatorname{Hr}^{r}\left(M ; Z_{2}\right)(r=0, \cdots, n)$ satisfying:

2. $U_{i}=W_{i}+\sum_{r=0}^{i-1} \mathrm{Sq}^{i-r}\left(U_{r}\right)$ where $U_{0}=W_{0}=1$ and ${ }^{n} \mathrm{Sq}^{n}$ is the Steenrod square [3].

3. $U_{r} P_{n-r}=\mathrm{Sq}^{r}\left(P_{n-r}\right)$ where $P_{n-r}$ is a homogeneous polynomial of degree $n-r$ in the Stiefel-Whitney classes.

The Steenrod squares satisfy the Cartan formula ([3]).

4. $\operatorname{Sq}^{r}\left(W_{p} W_{q}\right)=\sum_{a=0}^{r} \operatorname{Sq}^{a}\left(W_{p}\right) \operatorname{Sq}^{r-a}\left(W_{q}\right)$.

In $\mathrm{Wu}[7]$ it is shown that:

5. $\mathrm{Sq}^{r}\left(W_{i}\right)=\sum_{i=0}^{r} B(i-r+t, t) W_{r-t} W_{i+t}$ for $r \leqq i$ and zero otherwise. $B(X, t)$ is the binomial coefficient $\left(\frac{X-1}{t}\right) \bmod 2$ if $X-1 \geqq t$ and 0 otherwise.

For $X \geqq 1$ an easy computation shows that:

6. $B(X, 0)=1$ all integers $X$ and $B(X, i)=1$ if and only if $X \equiv 0$, $i+1 \bmod 4$ for $i=1,2,3$.

Let $P_{n-r}=W_{p} W_{q}$ in $3(p+q=n-r)$. A simple calculation using 1, $3,4,5$ yields:

7. If $n=p+q+r, 0<m=\min (p, r)$ and $r \leqq q$ then $\sum_{\varepsilon=0}^{m} B(p, a)$ $\cdot B(q, r-a) W(p+a)=0$.

LeMma 2. Let $M$ be an n-manifold with $C(M) \leqq 3$. If $n=4 s+3$ then:
a. $W(4 t+1)=0(0 \leqq t \leqq s-1)$
b. $W(4 t+2)=0(0 \leqq t \leqq s-1)$
c. $W(4 t)+W(4 t+1)=0(1 \leqq t \leqq s)$
d. $\sum_{i=0}^{3} W(4 t+i)=0(1 \leqq t \leqq s-1)$
e. $W(0)=0(s \geqq 0)$.

Proof of Lemma 2.

a. Let $r=2$ and $p=4 t+1$. The conditions on $n, t$ and $n=p+q+r$ mply $0<p \equiv 1$ and $0<q \equiv 0 \bmod 4$. The result follows from 6 and 7 .

b. Let $r=2$ and $p=4 t+2$. The conditions on $n$ and $t$ imply $0<p \equiv 2$ and $0<q \equiv 3 \bmod 4$. The result follows from 6 and 7 .

c. Let $r=1$ and $p=4 t$. The conditions on $n$ and $t$ imply $0<p \equiv 0$ and $0<q \equiv 2 \bmod 4.6$ and 7 give the result.

d. Let $r=3$ and $p=4 t$. The conditions on $n$ and $t$ imply $0<p \equiv 0$ and $0<q \equiv 0 \bmod 4.6$ and 7 give the result.

e. From $2, W_{0}=U_{0}=1$ and $W_{1}=U_{1}$. Letting $r=1$ and $P_{n-r}=W_{n-1}$ in 3 gives $W_{1} W_{n-1}=\mathrm{Sq}^{1}\left(W_{n-1}\right)$. Using 5 and 6 with $n-1 \equiv 2 \bmod 4$ gives $W(0)=0$. This proves Lemma 2 .

Let $s \geqq 1$. Letting $t=0$ in $a$ and $b, t=s$ and $W(i)=W(n-i)(i=1,2)$ in $c$, and $e$ yields:

8. $W(i)=0, i=0,1,2,3$. 
This proves Lemma 1 for $n=7$.

If $s \geqq 2$ then $t=1, \cdots, s-1$ in $a, b, c$ and $d$ gives:

9. $W(i)=0, i=4,5, \cdots, n-4$. For $n \geqq 11$ Lemma 1 follows from 8 and 9. This completes the proof of Lemma 1 and hence of the theorem.

\section{REFERENCES}

1. M. K. Fort, Jr. (Editor), Topology of 3-manifolds and related topics, PrenticeHall, Englewood Cliffs, N. J., 1962.

2. J. Milnor, Lectures on characteristic classes, Mimeographed Notes, Princeton University, 1957.

3. N. E. Steenrod, Cohomology operations, Ann. of Math. Studies, No. 50, Princeton Univ. Press, Princeton, N. J., 1962.

4. R. Thom, Quelques proprietes globales des varietés differentiables, Comment. Math. Helv. 28 (1954), 17-86.

5. A. H. Wallace, Modifications and cobounding manifolds, Canad. J. Math. 12 (1960), 503-528.

6. W. Wu, Classes caracteristiques et i-carres d'une variete, C. R. Acad. Sci. Paris 230 (1950), 508-511.

7. - Les i-carres dans une variete grassmannienne, C. R. Acad. Sci. Paris 230 (1950), 918-920.

UNIVERSITY OF MIAMI 